\title{
Discernibility Thresholds and Approximate Dependency in Analysis of Decision Tables
}

\author{
Yu-Ru Syau ${ }^{1}$ En-Bing Lin $^{2 *}$, Lixing Jia ${ }^{3}$ \\ ${ }^{1}$ Department of Information Management, National Formosa University, Yunlin, 63201, Taiwan. \\ 2 Department of Mathematics, Central Michigan University, Mt. Pleasant, Michigan 48859, USA. \\ ${ }^{3}$ Department of Mathematics \& Computer Science, Chicago State University, Chicago, IL 60628, USA. \\ *Corresponding author. Tel.: 9897743597; email: enbing.lin@cmich.edu \\ Manuscript submitted March 15, 2015; accepted August 2, 2015 \\ doi: $10.17706 /$ jcp.10.6.412-417
}

\begin{abstract}
The concept of discernibility thresholds in the variable precision model (VP-model) is presented to classify sets in a nonempty finite universe. Analysis of inconsistent decision table is obtained by using the tools in VP-model. The tools are obtained by the interplay among inclusion error, discernibility threshold dependency and consistency as well as lower and upper approximation with thresholds. We also provide the relationships between the inclusion errors and lower approximations with threshold.
\end{abstract}

Key words: Inconsistent decision tables, lower and upper approximations, discernibility, rough sets, variable precision rough set models.

\section{Introduction}

Pawlak proposed rough set theory (RST) [1] which is based on fundamental set theory and has been applied and extended in many different aspects of theoretical and applied research areas [2], [3], [4]. Using a table to represent some collection of data in the real world, on the other hand, considering the concepts of lower and upper approximations in rough set theory, one can derive some decision rules of the knowledge system which is described in an information table by using the tools obtained from RST. In order to analyze or classify the data more effectively and correctly, we use the concept of variable precision which was introduced by Ziarko [5]. In this paper, we first recall some background in RST and the concept of variable precision. We then use the notion of discernibility thresholds to classify sets in a given nonempty finite universe. We further improve a result of Ziarko [5], and conclude with formulas for approximate dependency of attributes of inconsistent decision tables.

\section{Preliminaries}

Let $U$ denote a nonempty finite set, called the universe, and $2^{U}$ the collection of all subsets of $U$, including the whole set $U$ and the empty set $\emptyset$. The Cartesian product $U \times U$ is the set of all ordered pairs of elements of $U$. Set inclusion is denoted by $\subseteq$, and strict set inclusion by $\subset$. The cardinality of a set $S \subseteq U$, denoted $|S|$, is the number of elements in $S$.

The greatest lower bound (infimum) and least upper bound (supremum) of a set $S$ of real numbers are denoted by inf $S$ and sup $S$, respectively. By convention,

$$
\inf \varnothing=+\infty \quad \text { and } \quad \sup \varnothing=-\infty \text {. }
$$


If inf $S \in S$ (respectively, $\sup S \in S$ ), then we also denote it by min $S$ (respectively, max $S$ ) and call it the minimum (respectively, the maximum) of $S$.

Let $R \subseteq U \times U$ be an equivalence relation. If $(x, y) \in R$, we say that $x$ and $y$ are equivalent to each other. The $R$-equivalence class of an element $x \in U$, denoted $[x]_{R}$, is the subset of all elements in $U$ which are equivalent to $x$. The collection $U / R$ of all distinct $R$-equivalence classes, called the quotient set of $U$ by $R$, forms a partition of $U$.

For nonempty subsets $X$ and $Y$ of $U$, according to Ziarko [5], the inclusion error of $X$ in $Y$, denoted by $e(X, Y)$, which is defined as

$$
e(X, Y)=1-\frac{|X \cap Y|}{|X|} .
$$

For $\beta \in[0,0.5)$, Ziarko [5] defined $X \subseteq^{\beta} Y$ by

$$
X \subseteq^{\beta} Y \text { if and only if } \quad e(X, Y) \leq \beta .
$$

\section{Lemma 1 [5]}

Let $\beta \in[0,0.5)$. If $A \cap B=\emptyset$ and $X \subseteq^{\beta} B$ then it is not true that $X \subseteq^{\beta} A$.

This gives rise to a natural description of inclusion by using inclusion error with a threshold.

\subsection{Variable Precision of Rough Sets}

Let $R \subseteq U \times U$ be a given equivalence relation. For any $X \subseteq U$, according to Pawlak [1], [2], a pair of lower and upper approximations, $\underline{R}(X)$ and $\bar{R}(X)$, respectively, of $X$ are defined as follows.

$$
\begin{gathered}
\underline{R}(X)=\bigcup\{E \in U / R \mid E \subseteq X\}, \\
\bar{R}(X)=\bigcup\{E \in U / R \mid E \cap X \neq \emptyset\} .
\end{gathered}
$$

By replacing " $\subseteq$ " with " $\subseteq \beta$ ", Ziarko [5] obtained the following generalized notion of $\beta$-lower approximation or $\beta$-positive region of $X$,

$$
\begin{gathered}
\underline{R}^{\beta}(X)=\bigcup\left\{E \in U / R \mid E \subseteq^{\beta} \quad X\right\}=\bigcup\{E \in U / R \mid e(E, X) \leq \beta\} \\
=\bigcup\left\{E \in U / R \mid \frac{|E \cap X|}{|E|} \geq 1-\beta\right\} .
\end{gathered}
$$

The $\beta$-upper approximation of $X$ is defined as

$$
\bar{R}^{\beta}(X)=\bigcup\{E \in U / R \mid e(E, X)<1-\beta\}=\bigcup\left\{E \in U / R \mid \frac{|E \cap X|}{|E|}>\beta\right\} .
$$

Rough set theory with such approximations is referred to as the variable precision rough set model (VP-model) [5]. The $\beta$-boundary region $B N R^{\beta}(X)$ of $X$, in the VP-model, is defined by

$$
B N R^{\beta}(X)=\bar{R}^{\beta}(X)-\underline{R}^{\beta}(X)=\cup\{E \in U / R \mid \beta<e(E, X)<1-\beta\} .
$$

The set $X$ is said to be $\beta$-discernable if $B N R^{\beta}(X)=\emptyset$, or equivalently, if $\underline{R}^{\beta}(X)=\bar{R}^{\beta}(X)$. The least value of inclusion error $\beta$ which makes $X$ discernable will be referred to as discernibility threshold.

A set which is not $\beta$-discernable for every $\beta \in[0,0.5)$ will be called absolutely indiscernible. There are several properties of lower approximation, upper approximation of certain thresholds and discernibility which are described as follows.

Lemma 2 [5] 
Let $R \subseteq U \times U$ be an equivalence relation, and let $\beta \in[0,0.5)$. Then

i) $\underline{R}^{\beta}(\varnothing)=\bar{R}^{\beta}(\varnothing)=\emptyset ; \underline{R}^{\beta}(U)=\bar{R}^{\beta}(U)=U$.

ii) $\bar{R}^{\beta}(X)=U-\underline{R}^{\beta}(U-X), \forall X \subseteq U$.

iii) for $\beta^{\prime} \in[\beta, 0.5)$, we have

$$
\underline{R}^{\beta}(X) \subseteq \underline{R}^{\beta^{\prime}}(X) \subseteq \bar{R}^{\beta^{\prime}}(X) \subseteq \bar{R}^{\beta}(X), \forall X \subseteq U .
$$

iv) $\underline{R}^{0}(X)=\underline{R}(X) ; \bar{R}^{0}(X)=\bar{R}(X), \forall X \subseteq U$.

\subsection{Decision Tables}

An information table is a 4-tuple $(U, A, V, f)$, where $U$ is a nonempty finite universe, $A$ is a nonempty finite set of attributes, $V$ is the union of attribute domains, and $f: U \times A \rightarrow V$ is an information function defined for every $x \in U$ and $a \in A$, such that $f(x, a) \in V_{a}$, where $V_{a}$ is a domain of the attribute $a$.

Each nonempty $B \subseteq A$ determines an equivalence relation $R_{B}$ on $U$ as follows.

$$
R_{B}=\{(x, y) \in U \times U \mid \forall a \in B, f(x, a)=f(y, a)\} .
$$

If we distinguish in an information table, two disjoint classes of attributes, called condition and decision attributes, respectively, then the information table will be called a decision table and denoted by $(U, C \cup D, V$, $f$ ), where $C$ and $D$ are disjoint sets of condition and decision attributes, respectively. The $R_{D}$-equivalence classes will be called decision classes.

A decision table $(U, C \cup D, V, f)$ is consistent, if and only if $R_{C} \subseteq R_{D}$; otherwise, the decision table is inconsistent.

\subsection{Approximate Dependency of Attributes}

Let $(U, C \cup D, V, f)$ be an inconsistent decision table. For each nonempty $P \subseteq C$, Ziarko [5] defined in VP-model the $\beta$-positive region of the partition $U / R_{D}$ with respect to (in short, w.r.t.) $P$, denoted $\operatorname{POS}^{\beta}(P$, $D$ ), and $\beta$-dependency level of decision attributes $D$ w.r.t. $P$, denoted $\gamma^{\beta}(P, D)$, by

$$
\begin{gathered}
\left.\operatorname{POS}^{\beta}(P, D)=\bigcup\left\{D_{j} \in U / R_{D} \mid D_{j} \subseteq^{\beta} R_{P}\right)\right\}, \\
\gamma^{\beta}(P, D)=\frac{\left|P O S^{\beta}(P, D)\right|}{|U|} .
\end{gathered}
$$

The value $\gamma^{\beta}(P, D)$ measures the relative size of objects in $U$ for which classification (based on $U / R_{D}$ ) is possible within the classification error $\beta$ [4], [5]. According to Ziarko [5], a $\beta$-reduct of the condition attributes $C$ w.r.t. to the decision attributes $D$ is a minimal subset $Q \subseteq C$ for which $\gamma^{\beta}(Q, D)=\gamma^{\beta}(C, D)$.

\section{Remarks on Discernibility Thresholds in the VP-Model}

Consider an equivalence relation $R$ on a nonempty finite universe $U$. According to (7), a set $X \subseteq U$ is absolutely indiscernible if and only if $e(E, X)=0.5$ for some $E \in U / R$. This suggests the following classification of sets in $U$ :

\section{Lemma 2}

$$
\begin{aligned}
& S(R, X)=U\{E \in U / R \mid e(E, X)<0.5\} \\
& M(R, X)=\bigcup\{E \in U / R \mid e(E, X)=0.5\} \\
& L(R, X)=U\{E \in U / R \mid e(E, X)>0.5\} .
\end{aligned}
$$


Let $R$ be an equivalence relation on a nonempty finite set $U$. For $X \subseteq U$, if

$$
S(R, X)=U\{E \in U / R \mid e(E, X)<0.5\}=\emptyset
$$

then $\underline{R}^{\beta}(X)=\emptyset$ for every $\beta \in[0,0.5)$.

\section{Example 1}

Considering a set $U=\left\{x_{1}, x_{2}, x_{3}, x_{4}, x_{5}, x_{6}\right\}$, let $R$ be the equivalence relation on $U$ corresponds to the partition $U / R=\left\{\left\{x_{1}, x_{2}, x_{3}\right\},\left\{x_{4}, x_{5}, x_{6}\right\}\right\}$.

Let us compute the inclusion errors of the $R$-equivalence classes

$$
E_{1}=\left\{x_{1}, x_{2}, x_{3}\right\}, \quad E_{2}=\left\{x_{4}, x_{5}, x_{6}\right\}
$$

in the set $X=\left\{x_{1}, x_{5}\right\}$. According to (1), we have

$$
e\left(E_{1}, X\right)=1-\frac{1}{3}=\frac{2}{3}, \quad e\left(E_{2}, X\right)=1-\frac{1}{3}=\frac{2}{3},
$$

which gives $S(R, X)=\emptyset, M(R, X)=\emptyset$, and $L(R, X)=U$. Since $S(R, X)=\emptyset$, it follows from (5) that for any $\beta \in[0,0.5), \underline{R}^{\beta}(X)=\emptyset$. This validates Lemma 2 .

Using (6) and (11), we obtain

$$
\bar{R}^{\beta}(X)=\varnothing \text { if and only if } \quad \beta \geq \frac{1}{3}
$$

which follows that

$$
\underline{R}^{\beta}(X)=\bar{R}^{\beta}(X)=\emptyset \text { for } \beta \geq \frac{1}{3} .
$$

Since inclusion errors are between 0 and 1, we normalize (1) as follows.

$$
\inf \emptyset=1 \quad \text { and } \quad \sup \emptyset=0
$$

We now improve Proposition 3.11 in Ziarko [5] as follows:

\section{Theorem 1}

Let $R$ be an equivalence relation on a nonempty set $U$. For $X \subseteq U$, let

$S(R, X)=U\{E \in U / R \mid e(E, X)<0.5\}$,

$M(R, X)=U\{E \in U / R \mid e(E, X)=0.5\}$,

$\lambda(R, X)=1-\inf \{e(E, X) \mid E \in U / R \& e(E, X)>0.5\}$, and

$\mu(R, X)=\sup \{e(E, X) \mid E \in U / R \quad \& \quad e(E, X)<0.5\}$.

1) If $M(R, X) \neq \emptyset$ then $X$ is absolutely indiscernible. Additionally, if $S(R, X) \neq \emptyset$ then $\mu(R, X)$ is the least value of inclusion error $\beta$ which results in the largest $\beta$-lower approximation $\underline{R}^{\beta}(X)$ of the set $X$.

2) If $M(R, X)=\emptyset$ then $X$ is discernable and its discernibility threshold is equal to the maximum of $\{\lambda(R, X), \mu(R, X)\}$, i.e.,

$$
\zeta(R, X)=\max \{\lambda(R, X), \mu(R, X)\} .
$$

Additionally, if $S(R, X)=\emptyset$ then we have

$$
\begin{gathered}
\underline{R}^{\beta}(X)=\emptyset \text { for every } \beta \in[0,0.5), \\
\mu(R, X)=0, \zeta(R, X)=\lambda(R, X), \text { and } \\
\bar{R}^{\beta}(X)=\emptyset \text { for } \beta \geq \zeta(R, X) .
\end{gathered}
$$




\section{Formulas for Approximate Dependency}

Let $(U, C \cup D, V, f)$ be an inconsistent decision table. The value $\gamma^{\beta}(C, D)$ measures the relative size of objects in $U$ for which classification (based on $U / R_{D}$ ) is possible within the classification error $\beta$. Accordingly, the value $\gamma^{\beta}(C, D)$ measures the ability to do the classification (the quality of classification) for specific value of $\beta$ [4], [5].

We denote

$$
U / R_{D}=\left\{D_{1}, D_{2}, \cdots, D_{k}\right\}
$$

For distinct decision classes $D_{i}$ and $D_{j}$ in $U / R_{D}$, according to (5) and Lemma 1, we have

$$
{\underline{R_{C}}}^{\beta}\left(D_{i}\right) \cap{\underline{R_{C}}}^{\beta}\left(D_{j}\right)=\emptyset .
$$

Therefore, from (9) and (10), we obtain

$$
\gamma^{\beta}(C, D)=\frac{\left|\operatorname{POS}^{\beta}(C, D)\right|}{|U|}=\sum_{\mathrm{j}=1,2, \ldots, k} \frac{\left|R_{C}^{\beta}\left(D_{j}\right)\right|}{|U|} .
$$

\section{VP-Model in Analysis of Inconsistent Decision Tables}

Theorem 1 together with (15) can be effectively applied to analysis of inconsistent decision tables. For a given inconsistent information system $(U, C \cup D, V, f)$, the procedure of the VP-model has three main steps and they are:

Step 1 Computing respectively all equivalence classes for $R_{C}$ and $R_{D}$ :

$$
U / R_{C}=\left\{E_{1}, E_{2}, \cdots, E_{l}\right\}, \quad U / R_{D}=\left\{D_{1}, D_{2}, \cdots, D_{k}\right\} .
$$

Step 2 For each decision class $D_{j}$,

1) computing each

$$
e\left(E_{i}, D_{j}\right) \text { for } j=1,2, \ldots, l .
$$

2) computing the following in several different cases,

$$
S\left(R_{C}, D_{j}\right), \quad M\left(R_{C}, D_{j}\right) \text { and } L\left(R_{C}, D_{j}\right) .
$$

i) If $S\left(R_{C} D_{j}\right) \neq \emptyset$, define

$$
\mu\left(R_{C}, D_{j}\right)=\max \left\{e\left(E_{i}, D_{j}\right) \mid E_{i} \in U / R_{C} \& e\left(E_{i}, D_{j}\right)<0.5\right\} ;
$$

otherwise $\mu\left(R_{C}, D_{j}\right)=0$.

ii) If $L\left(R_{C}, D_{j}\right) \neq \emptyset$, define

$$
\lambda\left(R_{C}, D_{j}\right)=1-\min \left\{e\left(E_{i}, D_{j}\right) \mid E_{i} \in U / R_{C} \& e\left(E_{i}, D_{j}\right)>0.5\right\} ;
$$

otherwise $\lambda\left(R_{C}, D_{j}\right)=0$.

iii) If $M\left(R_{C}, D_{j}\right)=\emptyset$, compute respectively

$$
\zeta\left(R_{C}, D_{j}\right)=\max \left\{\lambda\left(R_{C}, D_{j}\right), \mu\left(R_{C}, D_{j}\right)\right\} \text { and } \quad \gamma \zeta\left(R_{C}, D_{j}\right)(C, D) .
$$

iv) If $M\left(R_{C}, D_{j}\right) \neq \emptyset$ and $S\left(R_{C} D_{j},\right) \neq \emptyset$, compute $\gamma^{\mu\left(R_{C}, D_{j}\right)}(C, D)$.

v) If $M\left(R_{C}, D_{j}\right) \neq \emptyset$ and $S\left(R_{C}, D_{j}\right)=\emptyset$, we have

$$
{\underline{R_{C}}}^{\beta}\left(D_{j}\right)=\emptyset \text { for every } \beta \in[0,0.5) .
$$




$$
{\overline{R_{C}}}^{\beta}\left(D_{j}\right)=\emptyset \quad \text { for } \beta \geq \lambda\left(R_{C}, D_{j}\right)
$$

\section{Concluding Remark}

We have provided with an algorithm in calculating threshold dependency level of decision attributes with respect to condition attributes. This helps analyze inconsistent decision tables which would give rise to further applications in data analysis.

\section{Acknowledgment}

The authors would like to thank anonymous referees' helpful suggestions and comments that help us improve the paper.

\section{References}

[1] Pawlak, Z. (1982). Rough sets. International Journal of Computer and Information Science, 11, 341-356.

[2] Pawlak, Z. (1991). Rough sets: Theoretical Aspects of Reasoning about Data. Dordrecht: Kluwer Academic Publishers.

[3] Lin, T. Y. (1997). An overview of rough set theory from the point of view of relational databases. Bulletin of International Rough Set Society, 1, 30-34.

[4] Beynon, M. Reducts within the variable precision rough sets model: a further investigation. European Journal of Operational Research, 134(3), 592-605.

[5] Ziarko, W. (1993). Variable precision rough set model. Journal of Computer and System Sciences, 46, 39-59.

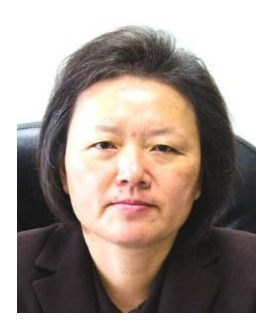

Yu-Ru Syau received her Ph.D. degree from the University of Illinois at Chicago, and now is the dean of the College of Management, a professor of information management at the National Formosa University, Taiwan. She also served as the chair of the Department of Information Management. Her teaching and research interests are in granular computing and the general areas of operations research.

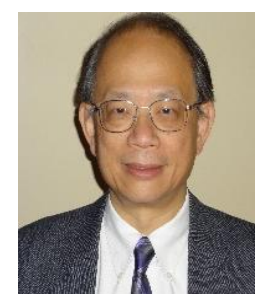

En-Bing Lin received his Ph.D. degree in mathematics from Johns Hopkins University. Currently, he is the chair and a professor of mathematics at Central Michigan University, USA. He has taught and visited at several institutions including Massachusetts Institute of Technology, University of Wisconsin-Milwaukee, University of California, Riverside, University of Toledo, UCLA, and University of Illinois at Chicago. His research interests include data analysis, image processing, applied and computational mathematics, wavelet analysis and applications, and mathematical physics. He has supervised a number of graduate and undergraduate students. He serves on the editorial boards of several mathematics journals and several academic committees of regional and national associations. He has organized several special sessions at regional IEEE conference and American mathematical society national and regional meetings.

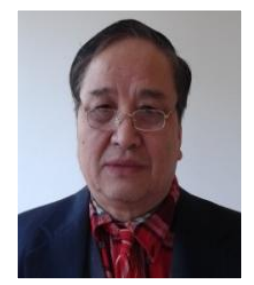

Lixing Jia received his Ph.D. degree in mathematics from the University of Illinois at Chicago. Currently he is a professor of mathematics \& computer science at Chicago State University, USA. He is specialized in applied mathematics. 